\title{
Gel Characteristics of Urea-Formaldehyde Resin under Shear Flow Conditions
}

\author{
Dang-liang Wang, Han-ying Bai, and Gao Yue \\ State Key Laboratory for Geomechanics and Deep Underground Engineering, China University of Mining and Technology, \\ Xuzhou 221008, China
}

Correspondence should be addressed to Dang-liang Wang; dlw9800@163.com

Received 18 March 2013; Revised 12 July 2013; Accepted 12 July 2013

Academic Editor: Waqar Khan

Copyright (C) 2013 Dang-liang Wang et al. This is an open access article distributed under the Creative Commons Attribution License, which permits unrestricted use, distribution, and reproduction in any medium, provided the original work is properly cited.

Urea-formaldehyde resin (UFR), one of chemical grouts in which the major ingredients are urea-formaldehyde and resin, is widely used in Chinese coal mines grouting. The gel characteristics of urea-formaldehyde resin (UFR) chemical grout under static conditions have been studied by many researchers. However, there is little research carried out on the gel characteristics under shear flow conditions. In fact, chemical grout like UFR keeps in shear flow conditions before gelling in the grouting process. In order to investigate the gel characteristics of UFR in shear flow conditions, an apparatus which consists of a magnetic stirrer and a viscometer was established. Magnetic stirrer was used to shear UFR at different velocity. Then the changes of UFR viscosity could be recorded by viscometer. As a result, the gel characteristics were summarized under different shear rates, and a formula of gelling is derived. The results show that the grouting flow rate influences the gelling time. Faster flow rates will cause longer gelling time, which means that the time for the grout to gel during the flowing process under shear flow conditions is longer than that under static conditions.

\section{Introduction}

Currently, chemical grout is widely used to control seepage and leakage, especially in the prevention and treatment of water and quicksand in coal mines. Urea-formaldehyde resin (UFR) has been used to address many difficulties as it has the advantages of low viscosity, good groutability, and good performance in terms of antiseepage and water plugging in a high pressure stratum. From 2002 to 2007, UFR resin chemical grout has been popularized and applied in the curing of shaft wall fractures and used in water and sand inrush disasters in the east of China. Aside from those advantages, UFR has provided remarkable economic benefits and social effects [1-4].

Typically, the gel time of grout under static conditions is set as a parameter that is used for reference when designing the grouting. However, the motion of grout has been overlooked. In reality, when underground water is in static conditions, the grout flow rate is lower, in which pressure is increased with time $[5,6]$. When underground water is in motion, the grout flow rate is faster, in which pressure is lower at first and increases after a lengthy amount of time [7, 8]. Regardless whether it is the grouting pressure in the pipeline or fractures and pore structures, it causes grout to remain in a flowing and shearing state. The gel time of grout under a flow state is the real gel time in real life practical situations, which has been neglected.

There is a national standard only for the testing of the gel time of epoxy resin grout. The method specified in this standard test the gel time of grout under static conditions. However, whether this standard can be employed for UFR still requires further studies [9]. Chen and Jiang analyzed a method to test the gel time of UFR in the early stages and argued that the inverted cup method is simple, practical, and convenient, which comprises the basis for recommendation in using the method [10]. However, this method lacks a set of full testing criteria. Furthermore, the method merely tests the gel time of grout under static conditions. In this paper, a set of equipment and techniques has been designed which can be applied to test the gelling of UFR under the state of shear and flow on the basis of the national standard that tests the gelling of epoxy resin. Moreover, this study aims to determine 
the flowing characteristics of the UFR chemical grout so as to draw the attention of experts in the field to this problem.

\section{Rheology of Chemical Grout}

2.1. Shear Thinning of Grout under Shear Flow Conditions. The gel time of chemical grout is an important parameter in the designing of chemical grouting. Currently, the testing methods adopted mainly include apparatuses such as a spreading Tu-four-cup or viscometer to measure the variation of the grout viscosity or strength under static conditions and determine the results of their parameters. In grouting practices, the grout gels are kept in motion rather than in static conditions. As a result, the gel time for grout under static conditions is not its real gel time.

High-molecular polymer grout is in a gelling state while flowing in a porous medium, in which the viscosity of the grout under the state of shear and flow is lower than that of grout under static conditions $[11,12]$.

\subsection{Relationship between Shearing Rate and Grout Viscosity.}

The viscosity of chemical grout has great variation between the impact of shearing motion and static conditions. As observed in previous research, most scholars have paid more attention to the grout viscosity or viscosity variation in gelling under static conditions. However, they have neglected the gel time of grout under a state of shear motion.

Research on the relationship between viscosity and shearing rate of normal fluids has involved three types of models: the Cross, Meter, and Karol models [13].

(1) Cross Model. The Cross model obtains the relation between viscosity and shearing rate through experiments that are conducted in an internal environment. The expression is given as follows:

$$
\mu=\mu_{\infty}+\frac{\mu_{0}-\mu_{\infty}}{1+\alpha \gamma^{n_{c}}}
$$

where $\mu_{\infty}$ refers to the fluid viscosity when the shearing rate is infinitely great, $\mu_{0}$ refers to the fluid viscosity when the shearing rate is zero, $\alpha$ is a constant, $\gamma$ is the shearing rate, and $n_{c}$ is the model index, $2 / 3$.

\section{(2) Meter Model. Consider}

$$
\mu=\mu_{\infty}+\frac{\mu_{0}-\mu_{\infty}}{1+\left(\gamma / \gamma_{1 / 2}\right)^{p-1}},
$$

where $p$ is the experience parameter and $\gamma_{1 / 2}$ refers to the shearing rate when the viscosity is $\mu_{0} / 2$.

\section{(3) Karol Model. Consider}

$$
\mu=\mu_{0}\left[1+(\lambda \gamma)^{2}\right]^{(n-1) / 2}
$$

where $t$ is the time constant and $n$ is the power law index or slope of the $\mu(\gamma)$ curve.

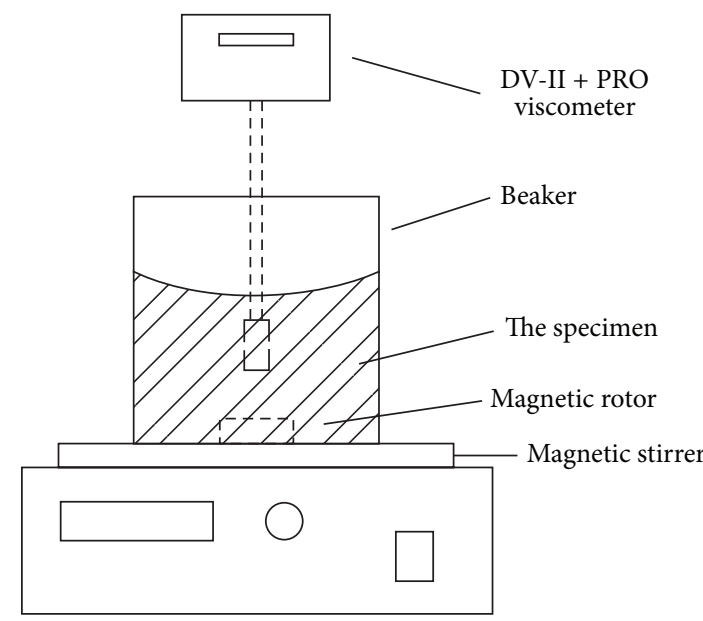

FIGURE 1: Testing device for gel characteristics.

TABLE 1: Gelling time for UFR in static conditions and motion (viscosity $100 \mathrm{mPa} \cdot \mathrm{s}$ for motion).

\begin{tabular}{lccccc}
\hline & \multirow{2}{*}{${\text { Ratios }(\mathrm{A}: \mathrm{B})^{*}}^{*}$} & \multicolumn{4}{c}{ Speed of rotor/rpm } \\
& & 0 & 987 & 1560 & 1980 \\
\hline \multirow{3}{*}{ Gel time/s } & $10: 1$ & 423 & 541 & 630 & 820 \\
& $10: 2$ & 190 & 350 & 460 & 542 \\
& $10: 4$ & 92 & 285 & 340 & 420 \\
\hline
\end{tabular}

${ }^{*}$ The concentration of $\mathrm{B}$ (acidity curing agent) is $8 \%$.

\section{Gel Characteristics and Formula of UFR Grout in Motion}

As grout flows under shearing conditions, it is relatively difficult to directly test the motion of grout gelling. In order to obtain the gelling properties of grout in motion, the experimental equipment shown in Figure 1 was designed for this purpose.

Testing device is composed of two components: a magnetic stirrer and a viscometer. The spinning of the rotor in the magnetic stirrer continuously moves the grout during the gelling process. Then, the variation of the grout viscosity in the gelling process is tested by using a DV-II+PRO on-line viscometer. At the same time, the spinning speed of the rotor in the magnetic stirrer has to be altered so as to change the speed of moving the grout.

3.1. Gel Time of UFR under Different Shear Rates. Experiments on grout gelling in static conditions and motion (rotor speed at 987 rounds per minute, rpm) were conducted, respectively, by using the experimental equipment as shown in Figure 1. The UFR that was used in this research comprises double liquids A and B. Liquid A is urea-formaldehyde and resin, while liquid $B$ is the acidity curing agent. The ratios of the double liquids in the UFR (A:B) were $10: 4,10: 2$, and $10: 1$. The testing results are shown in Table 1.

As shown in Figures 2(a) to 2(c), the gelling time of grout in motion is evidently lagging behind that of grout in static conditions. Furthermore, the less gelling time that the grout 


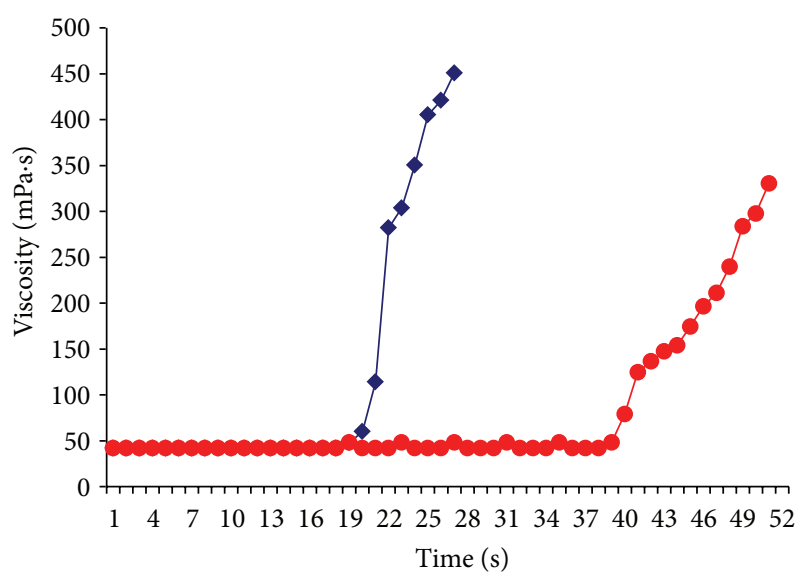

(a) Time-viscosity curve (ratio $\mathrm{A}: \mathrm{B}=10: 4$ )

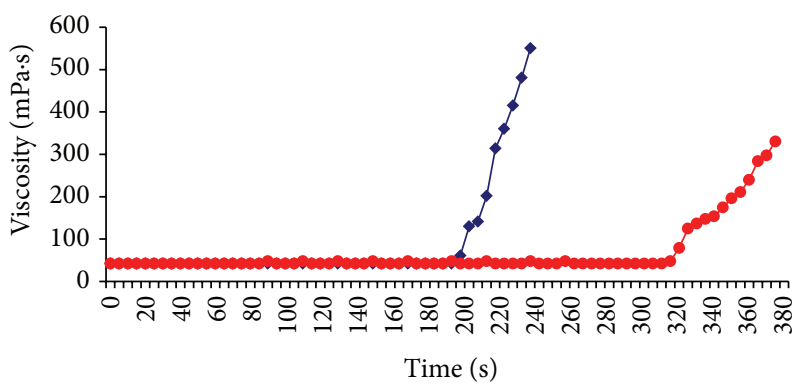

(b) Time-viscosity curve (ratio $\mathrm{A}: \mathrm{B}=10: 2$ )

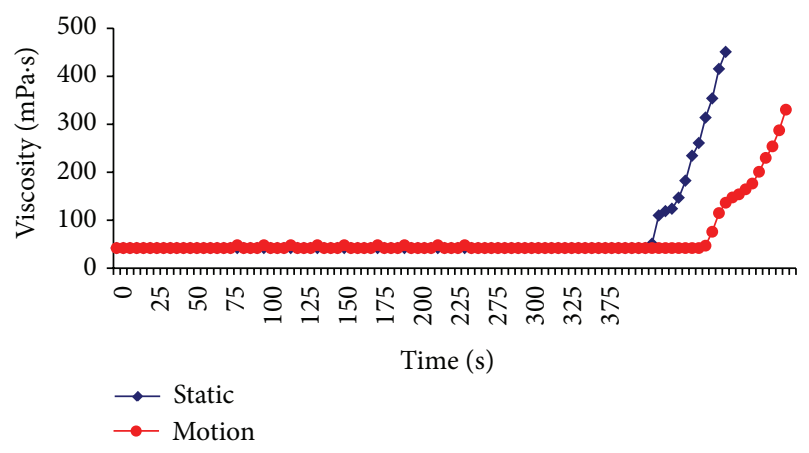

(c) Time-viscosity curve (ratio A: $\mathrm{B}=10: 1$ )

FIGURE 2: Time-viscosity curves for different ratios of UFR.

experiences, the more obvious the lag effect of the grout gelling in motion. As the amount of curing agent $\mathrm{B}$ that is added increases, both in static and dynamic environments, the gelling time is reduced, but in a more dynamic environment, this is even more so significantly reduced. Thus, the variation of the gelling characteristics under a state of shear and flow needs to be taken into account when designing the grouting.

\subsection{Comparison of Gel Time of UFR under Different Rotational} Speeds. In order to study the impact of the rotational speed of grout on the gel time, a comparison for the variation in the gelation of grout with a ratio of $10: 4$ at 987,1560 , and 1980 rpm was made, as shown in Figure 3.

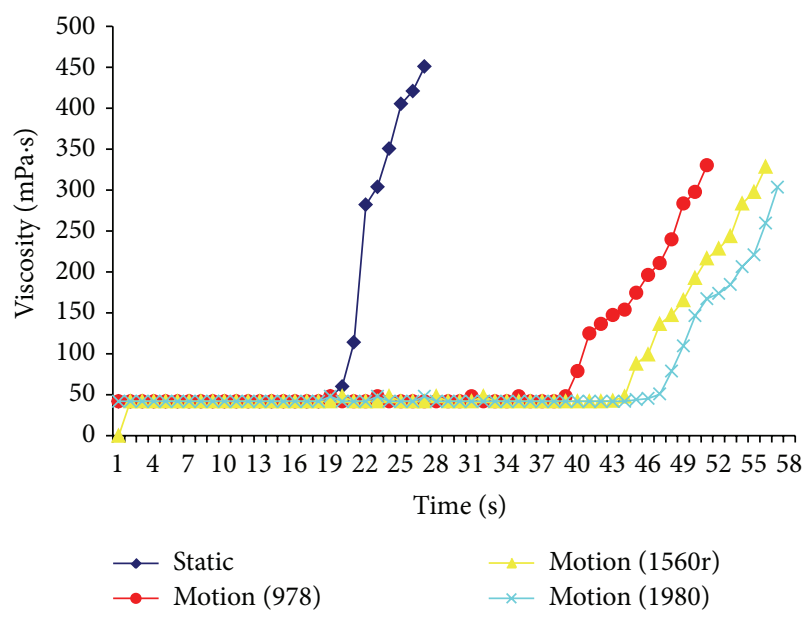

FIgure 3: Time-viscosity curve for different rotational speeds (ratio: $10: 4)$.

As shown in Figure 3, when grout is in a static condition, its gel time is about $100 \mathrm{~s}$. When the grout is stirred at rotational speeds of 987,1560 , and $1980 \mathrm{rpm}$ via a magnetic stirrer, its gel time is somewhere between 200 and $300 \mathrm{~s}$. In other words, the time of gelling while in motion is two to three times that of gelling under a static state. The experimental results indicate that a higher rotational speed means a more obvious impact on the grout by the shearing motion and a longer gel time of the grout Otherwise, a lower rotational speed means a less obvious impact on the grout, and a shorter gel time.

3.3. Mathematical Formula for Gelling of UFR. In view of $\tau$ as the viscosity resistance of fluid, a high polymer chemical grout used as the viscous fluid is Bingham fluid, which has a viscosity that increases with time. Therefore, the velocity and acceleration at a fixed point are related to the location of the fluid. However, fluid needs a certain time $t$ to flow from the grouting point to reach the fixed point. During period $t$, the grout fluid viscosity involves the following variations:

$$
\mu(t)= \begin{cases}\mu_{0}, & (t=0), \\ \mu(t), & (0<t<T), \\ \infty, & (t \geq T) .\end{cases}
$$

The graphic in Figure 3 is similar to a tangent function. Thus, the relation between $\mu(t) \sim t$ can be shown in the following equation:

$$
\mu(t)=\mu_{0}+\tan \left(\frac{t}{2 T} \pi\right) \quad(0<t<T),
$$

where $\mu_{0}$ refers to the viscosity of the grout fluid at the initial time and $T$ refers to the standard time for the gelling of the fluid grout relevant to its properties.

If the lag effect of the gel time of the grout in motion is taken into consideration, namely, the shear thinning behavior of grout, then the viscosity variation curve of chemical grout under a state of shear is shown in Figure 4. 


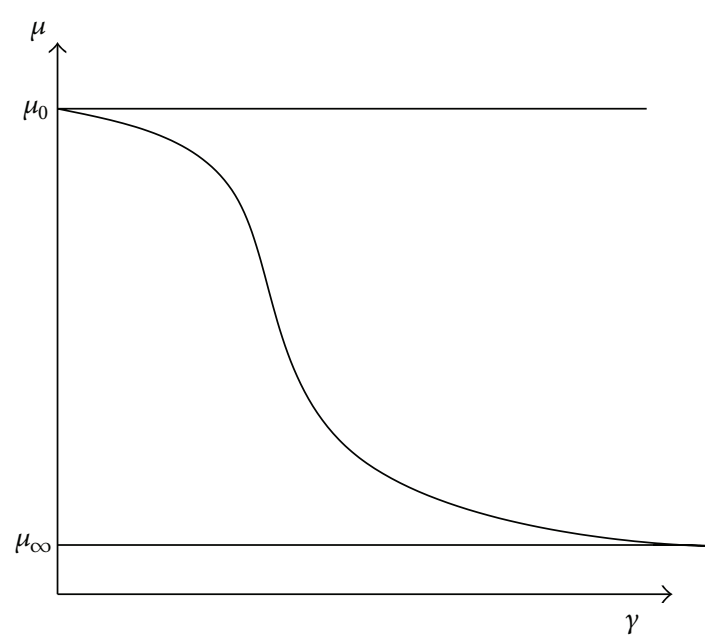

FIgURE 4: Typical viscosity-time curve of chemical grout in motion under shear flow conditions.

Based on the relation between the viscosity of the liquid grout and the shearing rate, it can be seen that the Cross model is a simple means and has a physically applicable format which not only describes the rheological behavior of the power law at high shearing rates, but also represents Newton rheological behavior that is close to a zero shearing rate. Consider

$$
\begin{gathered}
\mu(\gamma)= \begin{cases}\mu_{0}, & (\gamma=0), \\
\mu(\gamma), & (0<\gamma<\infty), \\
\mu_{\infty}, & (\gamma \longrightarrow \infty),\end{cases} \\
\mu=\mu_{\infty}+\frac{\mu_{0}-\mu_{\infty}}{1+\alpha \gamma^{n_{c}}}
\end{gathered}
$$

where $\mu_{0}$ refers to the grout viscosity when the shearing rate is zero. $\mu_{\infty}$ refers to the grout viscosity when the shearing rate is $\infty . \alpha$ is a constant. $\gamma$ is the shearing rate. $n_{c}$ is the model index, $2 / 3$.

Therefore, viscosity of grout in motion versus change in time should satisfy the equation:

$$
\mu(\gamma, t)=f(\gamma, t=0) \lambda(t),
$$

where $f(\gamma, t=0)$ refers to the function relationship between grout viscosity and shearing rate at the moment of zero; $\lambda(t)$ refers to the time correction factor at the moment of $t$ :

$$
0 \leq t \leq T, \quad \lambda(t)=\frac{\mu(t)}{\mu_{0}} .
$$

Thus, (8) is the mathematical equation for the gelling of UFR chemical grout.

The mathematical equation curve for the gelling of UFR chemical grout was calculated by applying MATLAB. As shown in Figure 5, as the shearing rate improves, the grout viscosity decreases and its frictional resistance also decreases, and the gel time is longer. Furthermore, the grout viscosity increases steadily and gradually becomes a gel with time.

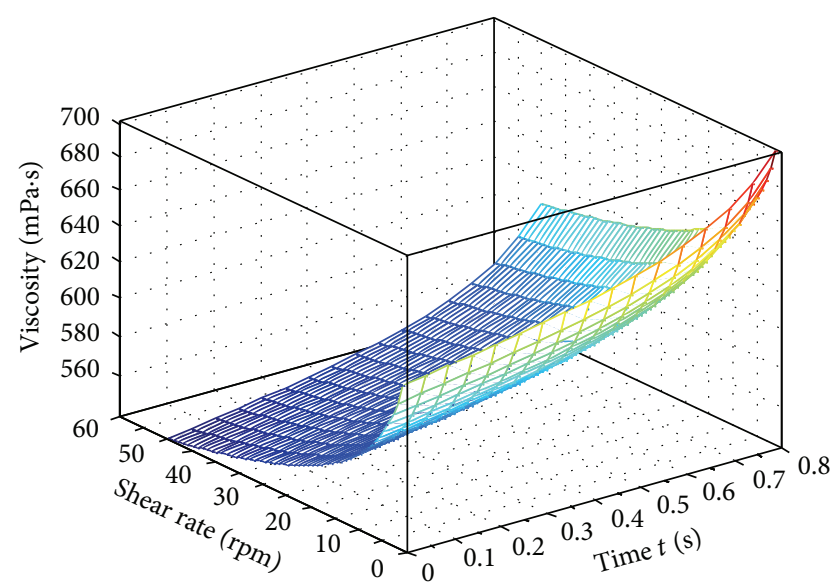

FIGURE 5: Mathematical curve for UFR gelling.

\section{Conclusion}

The variation laws of grout viscosity under different shearing rates by using a gel testing apparatus are examined in this paper by which a dynamic equation for the gelling of UFR grout in motion is established. The results of the equation calculations show that as the shearing rate improves grout viscosity decreases and its frictional resistance also decreases with a longer gel time. In other words, the gel time of grout in motion is longer than that of grout in a static state. This law should be taken into consideration in the designing of grouting.

\section{Acknowledgments}

Financial supports from National Natural Science Foundation under Grant no. 40772192 and Jiangsu Provincial Preponderant Discipline Construction Project are gratefully acknowledged.

\section{References}

[1] W. H. Sui, Z. Q. Jiang, and D. L. Wang, "One kind of chemical grouting material in coal mines: China," 200810123239. 2[P], 2008.

[2] D. L. Wang, W. H. Sui, and Z. Q. Jiang, "One kind of grouting material used in broken coal body solidification: China," 201110376459. 8[P], 2011.

[3] X. H. Ni, W. H. Sui, Y. Z. Guan et al., Study of Prevention Technology of Vertical Shaft Broken Borehole Wall in Coal Mines, Press of China University of Mining and Technology, Xuzhou, China, 2005.

[4] W. H. Sui, G. L. Zhang, Z. Q. Jiang, and D. L. Wang, "The stateof-the-art of chemical grouting treatment for quicksand hazards in coal mines and the prospect of several key problems," Journal of Engineering Geology, vol. 16, no. 1, pp. 73-77, 2008.

[5] D. L. Wang and W. H. Sui, "Research on law of grouting pressure change in rock mass," Metal Mine, no. 1, pp. 53-55, 2008.

[6] D. Wang and Y. Gao, "Empirical formula of chemical grouting pressure in porous media based on similarity theory," Applied Mechanics and Materials, vol. 71-78, pp. 3320-3324, 2011. 
[7] K. C. Ren, "Diffusing and grouting method of chemical grout with flowing water," Water Resources and Hydropower Engineering, no. 7, pp. 57-62, 1982.

[8] D. L. Wang, X. F. Dong, and W. D. Du, "Comparison analysis on pre-grouting experiment plan of high pressurized ultra large aquifer for coal mining face in shaft mine," Coal Engineering, no. 11, pp. 25-28, 2010.

[9] PRC Industrial Standard Editorial Group, GB12007. 7-89 Testing Method of Gelling Time of Epoxy Resin, China Standards Press, Beijing, China, 1990.

[10] L. H. Chen and Y. S. Jiang, "Determination of gel time of chemical grout," Railway Construction Technology, vol. 9, pp. 77-79, 2011.

[11] M. M. Cross, "Relation between viscoelasticity and shearthinning behaviour in liquids," Rheologica Acta, vol. 18, no. 5, pp. 609-614, 1979.

[12] A. Chougnet, A. Audibert, and M. Moan, "Linear and nonlinear rheological behaviour of cement and silica suspensions. Effect of polymer addition," Rheologica Acta, vol. 46, no. 6, pp. 793-802, 2007.

[13] T. Shi and D. F. Wu, Polymer Rheology Principles, Chemical Industry Press, Beijing, China, 2009. 


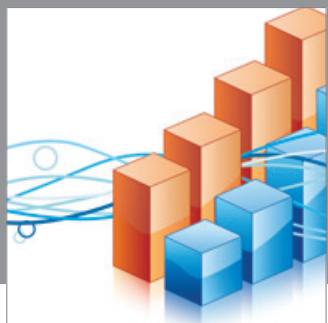

Advances in

Operations Research

mansans

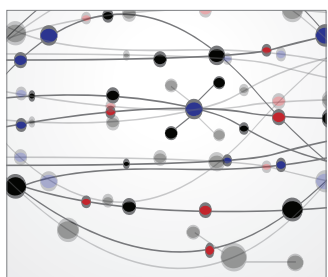

The Scientific World Journal
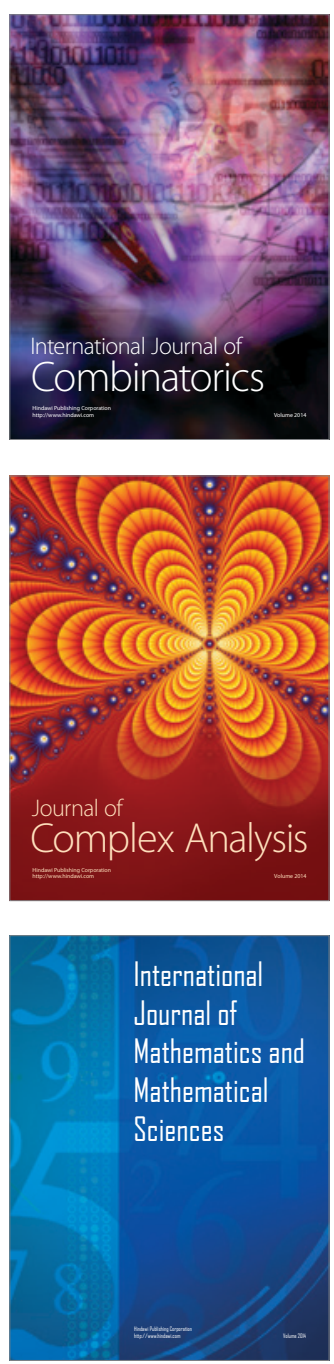
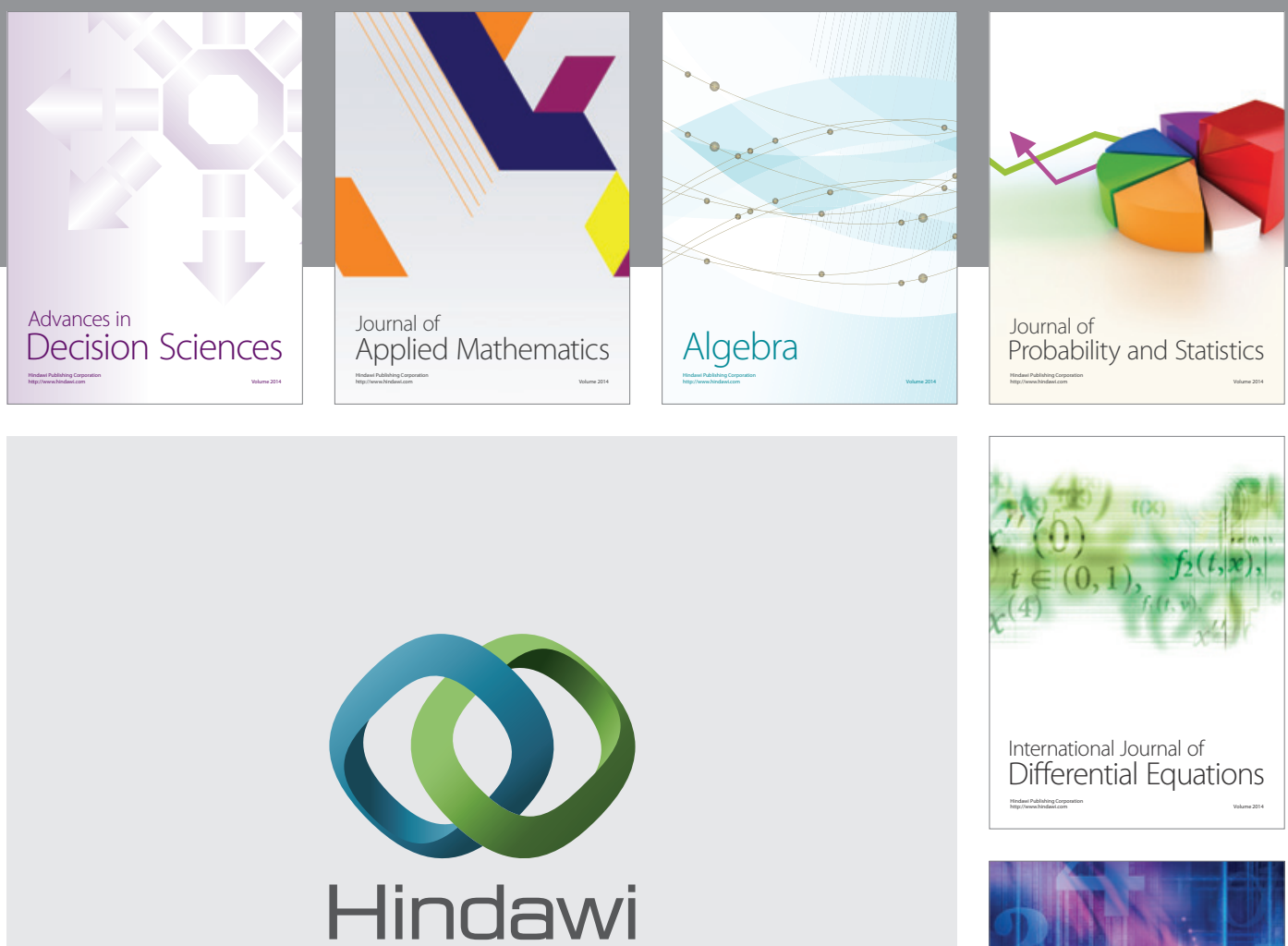

Submit your manuscripts at http://www.hindawi.com
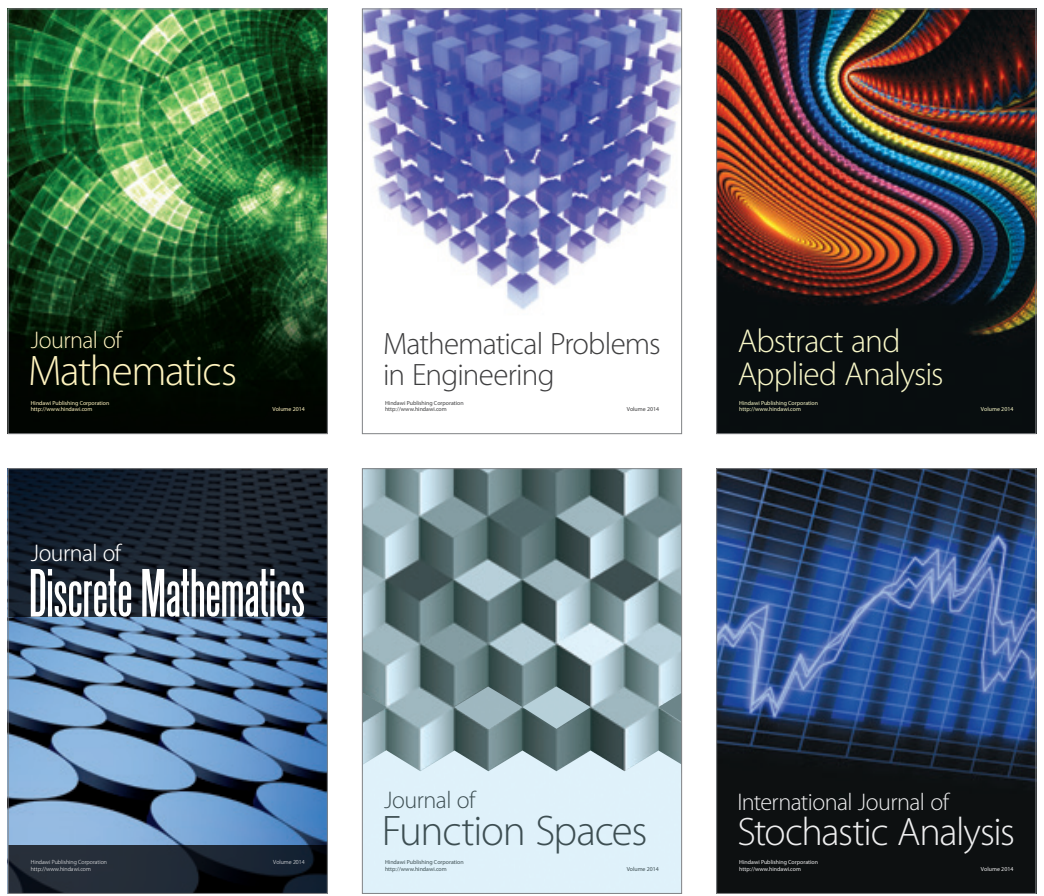

Journal of

Function Spaces

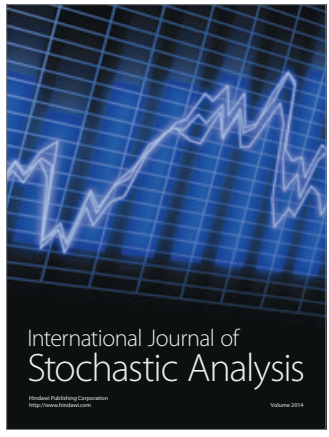

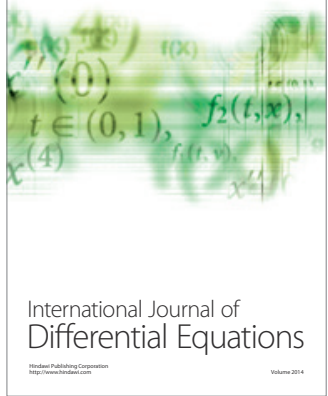
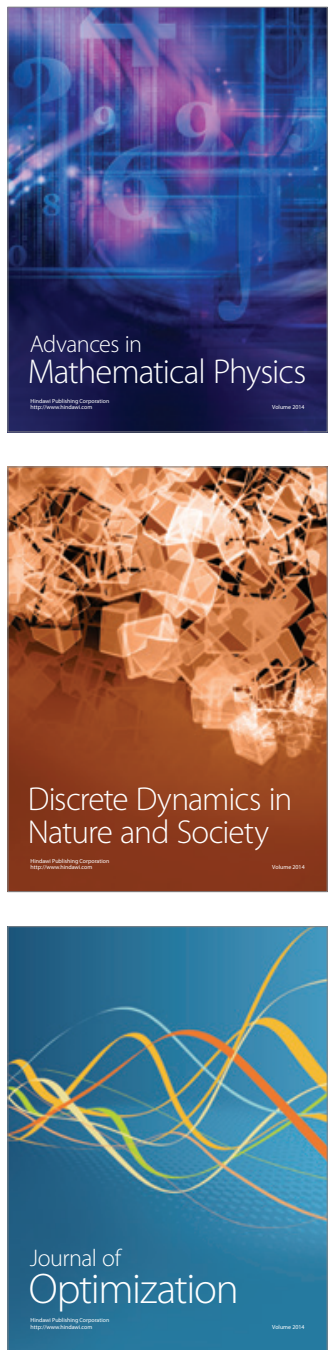